\title{
Innovative Batik Design with an Interactive Evolutionary Art System
}

\author{
Yang $\mathrm{Li}^{1,2}$ (李 扬), Chang-Jun $\mathrm{Hu}^{1}$ (胡长军), and Xin Yao (姚 新) ${ }^{2}$, Fellow, IEEE \\ ${ }^{1}$ School of Information Engineering, University of Science and Technology Beijing, Beijing 100083, China \\ ${ }^{2}$ The Centre of Excellence for Research in Computational Intelligence and Applications (CERCIA) \\ School of Computer Science, University of Birmingham, Edgbaston, Birmingham B15 2TT, U.K.
}

E-mail: y.li@cs.bham.ac.uk; huchangjun@ies.ustb.edu.cn; x.yao@cs.bham.ac.uk

Received March 18, 2009; revised July 14, 2009.

\begin{abstract}
This paper describes an evolutionary art system, which explores the potential ability of evolutionary computation in Batik design. We investigate the use of Interactive Evolutionary Algorithm (IEA) in our system, with the goal of enhancing user's creativity to generate innovative Batik-like patterns. We focus mainly on two crucial aspects of the system. First, a new representation is proposed to capture the features in Batik and create innovative patterns through evolutionary processes. Second, an out-breeding mechanism is applied to our system, in order to sustain user's interest for a longer period. Our system can search a much larger design space than other systems and can avoid being trapped in a local optimum. We describe the system in detail and the methodology we have adopted in the system. Our experimental results have shown that our newly developed system is effective and has great potentials in evolving novel Batik design.
\end{abstract}

Keywords Batik design, evolutionary art system, interactive evolutionary computation

\section{Introduction}

Evolutionary Art Systems (EASs) have been gaining popularity in designing innovative artworks for many years ${ }^{[1]}$. They are developed by combining the ideas of Evolutionary Computation (EC), and design theories to evolve images (and other artifacts) and generate aesthetic artworks ${ }^{[2]}$. In a number of real world applications, EASs have been developed to facilitate the designers' activities. However, there are no tools that can help to design Batik, which is a traditional art in Indonesia and southeast Asia.

Nowadays, Batik is used not only as fashion design for human clothes, but also as furnishing fabrics and household accessories. Batik is used by artists to create paintings which grace rooms and offices. However, fine quality handmade Batik is very expensive and the production of such works is very limited ${ }^{[3]}$. It is very interesting to investigate whether a computer-based Batik design system can be developed. EASs have been shown to be able to help different people to design exclusive patterns for practical applications according to their own preferences ${ }^{[4]}$. This paper describes a novel Batik design system based on Interactive Evolutionary Algorithm (IEA). Experimental studies have been carried out to demonstrate the effectiveness of the system in evolving highly innovative Batik patterns.

Existing EASs suffer from at least two major difficulties. First, the lack of explicit correlation between genotypes and phenotypes often limits effective evolution of artefacts. Second, the design space is often limited and fixed by a chosen representation. This paper will propose new techniques to solve these two issues.

In addition to the above, existing EASs also have the following unresolved issues in the design process ${ }^{[4]}$ :

1) Representation: images can be greatly varied and heavily relied on representations ${ }^{[2,5]}$. But it is always difficult to find an efficient way to capture the features in images without introducing redundancy. An inappropriate mapping from genotype to phenotype can lead to blind exploration in the evolutionary process.

2) User fatigue: it is important for EASs to prevent stagnation in evolution and keep users' interests high. Most systems use Interactive Evolutionary Computation (IEC), which is based on subjective human evaluation ${ }^{[6-9]}$. However, existing interactive EASs take a long time to find interesting images. So users usually lost interest in exploring the design space further.

Considering the above main issues, our system will aim at overcoming the drawbacks of existing EASs and producing innovative Batik pattern effectively through

\footnotetext{
Regular Paper

The first author was supported by the China Scholarship Council for sponsoring her work at the University of Birmingham, UK. This work was done while the first author was visiting the Centre of Excellence for Research in Computational Intelligence and Applications (CERCIA) at the University of Birmingham, UK.
} 
interactive evolution. This goal is achieved by (a) devising a new representation for various Batik styles, and (b) establishing a novel out-breeding mechanism that could apply more attractive patterns continuously and guide the evolutionary process towards more interesting solutions.

Three novel contributions are made by our work. First, a new on-line computer-aided system has been built for designing innovative Batik patterns. Second, both classical and innovative Batik patterns can be produced by our system effectively. Third, the system is user-friendly and very easy to use.

The rest of this paper is structured as follows. Section 2 begins with related work of EASs, followed by the description of our new representation in Section 3. Then in Section 4 a novel out-breeding mechanism is introduced to our system. A global overview of the system is given in Section 5. Some experimental results and their analysis are presented in Section 6. Finally, in Section 7 we draw some conclusions and point out directions for future research.

\section{Related Work}

The key ingredients of a successful implementation of EASs include the design problem, the representation and the search algorithm. Once the problem has been defined, the representation is considered as an important factor, the expressive power of which could affect the degree of exploring creative design by evolutionary algorithms. The representation used in evolutionary design can be classified into three categories according to the encoding methods ${ }^{[10]}$.

First, parameterization is a very traditional representation, which sufficiently describes the fixed topology of a design. Dawkins showed his work "Biomorphs", which could generate images by encoding a vector of sixteen real values ${ }^{[11]}$. Although this kind of representation is easy to design and fine-tune, not all designs can be encoded by parameters. Novel designs are hard to find due to the fixed design space.

Second, non-generative representation uses nonreusable rules to construct designs. The examples include L-systems ${ }^{[12]}$ and shape grammars ${ }^{[2]}$. Because of the exponential growth in the size of the design space, the distance between the initial space and the optimized design space increases. As the number of rules increases, search algorithms will require more steps to find a good solution. And due to the lack of correlations in the encoding rules, it requires more related rules to change simultaneously when only one rule changes. Therefore, non-generative representation is not well suited to handle the increasing size and the complexity in the design space.
Third, the ability of genetic representation of reusing elements or rules improves the search efficiency in large design space as well as the scalability by capturing design dependencies ${ }^{[13]}$. Sims created computer images using Lisp expressions to specify the color of every pixel ${ }^{[6]}$. In these expressions, the standard mathematical functions such as trigonometric, fractal functions and basic arithmetic operations were used. The design space defined by this representation is extremely large even with a limited number of expressions.

Later, Rooke ${ }^{[14]}$ extended this approach. He added iterative functions to increase the range of expressions. The output from his program is interpreted by a color mapping function that maps the scalar values to RGB vectors. There is no limit to the size of the design space (other than computer memory). Highly complex trees can be evolved.

Machado developed an evolutionary art program called NEvAr (Neural Evolutionary Art) ${ }^{[15]}$. By only using a set of simple functions, this representation is in contrast to Sim's genetic art that used a very rich function set. Unfortunately it also increases the time to breed "good" images.

Another work is Picbreeder, a popular online service, which allows users to collaboratively evolve images ${ }^{[16]}$. This on-line project uses Compositional Pattern Producing Networks (CPPNs) to produce patterns by composing functions. A CPPN graph topology is used to represent a large and diverse space of patterns, biased by the set of canonical functions.

However, for most of the EASs using Genetic Programming (GP), firstly, only abstract and simple images are generated from the random initial populations, which could not embody certain features in a specific design problem. It is difficult to find the genotypes that correspond to the existing patterns. Secondly, the search space is immense, so it takes a very long time to find the optimum. Thirdly, because of the lack of correlation between variation at the genotype level and that at the phenotype level, the fitness defined by such a problem is usually very rugged and difficult to improve.

This paper will address the above problems. Initial populations are randomly chosen from the space of classical Batik patterns. By using our representation, it is easier to explore the paths in the phenotype space according to the variations in the genotype space. The ability of exploring a variable design space is enhanced by a novel out-breeding mechanism. As a result, innovative Batik patterns can be continuously evolved using our system.

\section{Representation}

Our representation is created for generating Batik 
patterns. One of the fascinating characteristics of Batik is its rich variations in style and motif. We deeply explored the geometrical patterns and classified them into categories, establishing a fundamental description to present them.

\subsection{Our Approach}

Based on a multitude of collections in Batik, we find that the most common features are repetition and certain geometric transformations like rotation, translation, reflection, etc. These traits can be used to measure redundancy in these patterns. So, several minimal and sufficient sets of non-redundant primitives, together with the transformations, can be defined as the genotype.

Our representation is based on features, not pixels, of Batik patterns. The most basic elements of Batik features include triangle, polygon, circle, dot, star and flower patterns. These geometric patterns are a harmonious fusion of mathematics, art, and expressions of symmetry, balance, and ingenuity ${ }^{[3]}$. Each feature is generated from one gene in the genotype, and the Batik pattern embodies different features, as such the genotype is composed of different genes.

A genotype, $G(i)$, showed in Fig. 1 consists of a number of genes, each of which represents one feature in phenotype. Every gene has two evolvable attributes. The first part, unit set, is a map of points and edges, which represents the minimal sets before transforming into a feature; the second part, transformation, is a vector of matrix, each of which represents a transform of the set. The matrix is encoded in six alleles $a, b, c, d, e, f$, which is a two-dimensional $3 \times 3$ affine transform matrix, representing the $2 \mathrm{D}$ linear transform $((a b)(d e))$ together with the translation $(c f)$.

This representation is very simple, and easy to manipulate. Its length can be extended easily by adding in additional genes. Another advantage of this representation is that it compresses a pattern into a compact set of numbers. The pattern is made up of several unions of sets, each of which is transformed by the vector of transform matrix. An example of applying a condensation set to the transformation is illustrated in Fig.2. The unit set is first transformed by the matrix, the resulting flower-like pattern is then generated from the genotype.

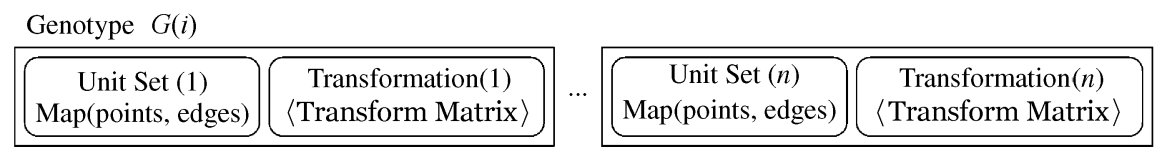

Fig.1. Genotype of $G(i)$.

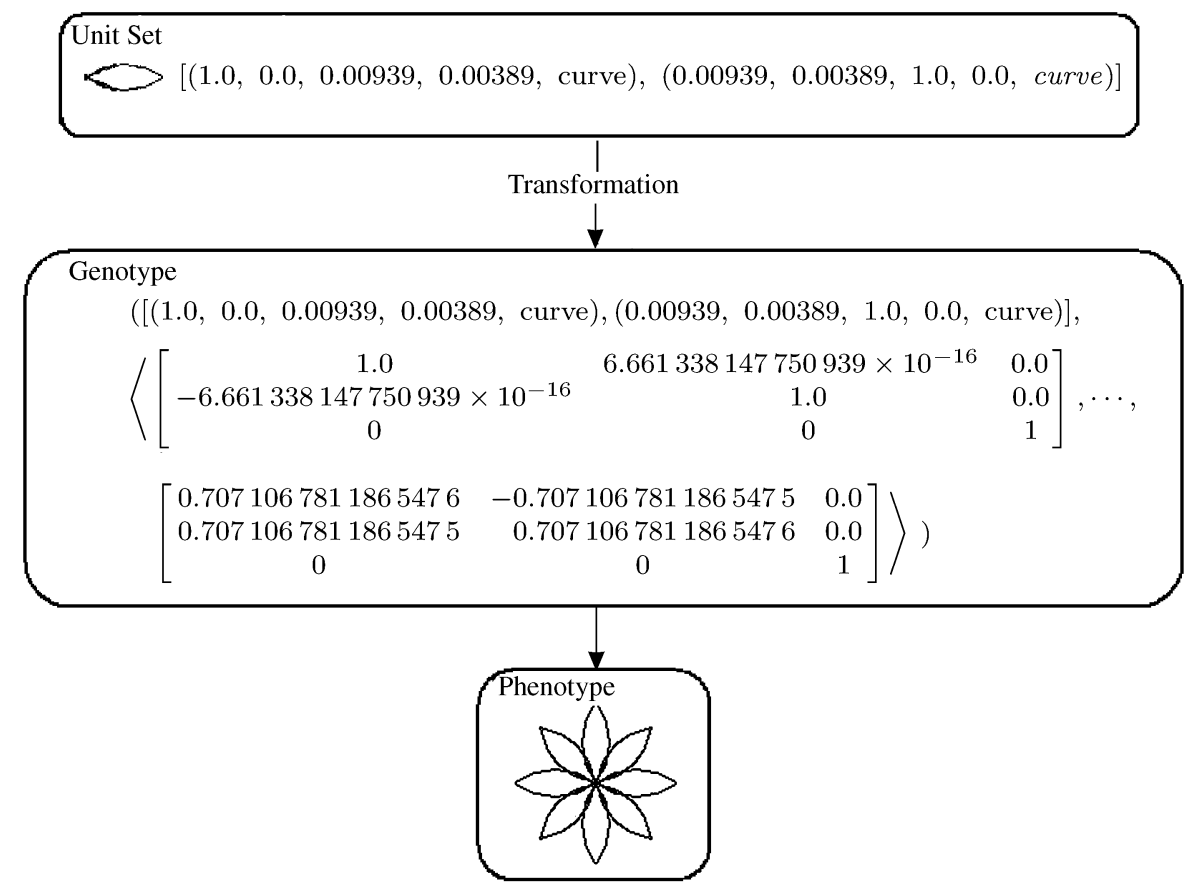

Fig.2. Example of mapping from genotype to phenotype. 


\subsection{Comparison with GP}

Since GP is the most commonly used scheme in EASs, two experiments are set up to compare our representation with GP in designing Batik.

First experiment applies GP as the representation. Table 1 shows the parameter setting for this experiment. Fig. 3 shows the initial populations represented by GP, which uses random functions and values to construct trees. Obviously they are quite simple and abstract. In order to generate a Batik pattern with the feature of circles and dots, 21 generations are used to produce the pattern in Fig.4. The corresponding genotype is $\max (x, \ln (\operatorname{sub}(\min (x, x),-0.2976)))$. We discovered that circles are shown since 20th generation.

Table 1. Parameters for GP Representation

\begin{tabular}{ll}
\hline Parameter & Setting \\
\hline Population size per generation & 18 \\
Number of generation & 21 \\
Mutation rate & 0.5 \\
Unary function set & sin, cos, tan, sqrt, abs, \\
& sqr, ln, exp, minus \\
Binary function set & $+,-, *, /$, max, min \\
Terminal set & $X, Y$ and random constants \\
Initial maximum tree depth & 6 \\
\hline
\end{tabular}

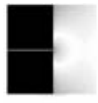

||
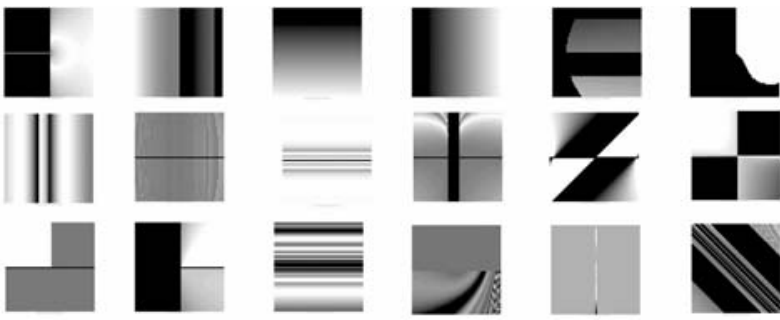

Fig.3. Initial populations represented by GP.

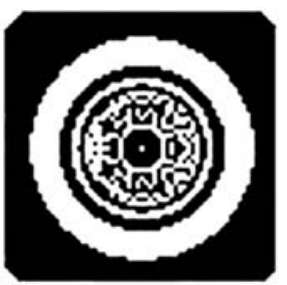

Fig.4. Batik-like pattern produced in the 21st generation by GP.

Our representation is used in the second experiment, parameter settings are shown in Table 2. We use Gene Generator (see Subsection 5.2) to initialize classical features in Batik patterns. The advantage for doing so is to constrain our initial design space to Batik patterns instead of starting from random patterns. Initial populations are shown in Fig.5. Most features are abstracted from traditional designs, which are represented by different genes to compose the corresponding genotype. The Batik pattern (see Fig.6) that was generated in the 5 th generation also includes the features of circles and dots, while the genotype contains only two genes.

Table 2. Parameters for Our Representation

\begin{tabular}{ll}
\hline Parameter & Setting \\
\hline Population size per generation & 8 \\
Number of generation & 5 \\
Number of genes in gene pool & 12 \\
Initial maximum genes & 3 \\
Crossover operators & Gene swap and \\
& gene replacement \\
\hline
\end{tabular}

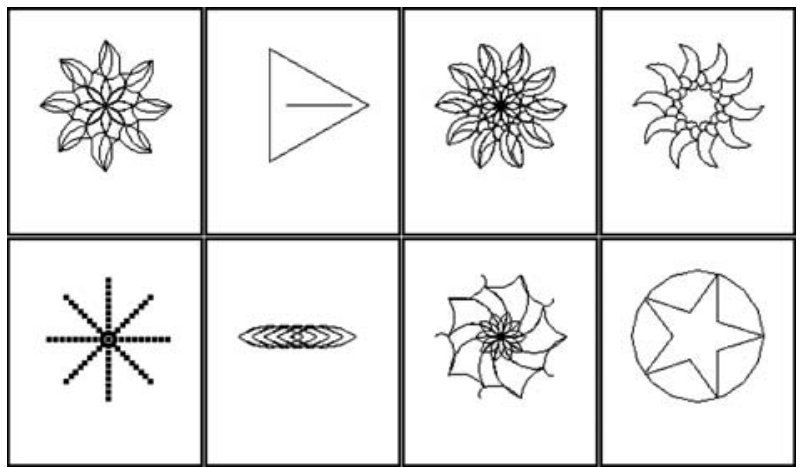

Fig.5. Initial populations represented by our system.

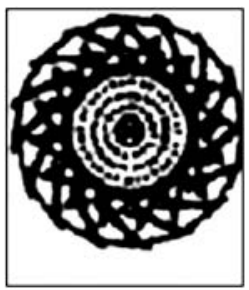

Fig.6. Batik-like pattern produced in the 5th generation by our system.

Although more complex functions could be used by GP for initial populations to produce Batik-like patterns, it takes much longer time to find a good solution in the large space. Our representation is more likely to generate innovative Batik patterns efficiently.

\section{Out-Breeding Mechanism}

Human beings easily lose their concentration on and interest in generating images in front of a computer, because EASs usually produce images that are quite similar to each other. In many cases, it takes a long time to find interesting images. In order to increase the diversity of solutions and prevent stagnation in evolution, we propose a novel out-breeding mechanism in the evolutionary process. 
The key idea behind out-breeding is to maintain two separate populations during interactive evolution, which are displayed as two separate panels on the computer screen. The first population evolves, based on the user's feedback, towards individuals (i.e., Batik patterns in our case) that are similar to the user's preferences, in order to find Batik patterns that the user likes. The second population evolves towards individuals that are most dissimilar to the user's preferences, in order to maintain sufficient diversity in exploring the design space. Whenever the first population is perceived to be trapped in a local optimum, individuals in the second population will be introduced to the first, so that a very different part of the design space can be explored.

Our current approach to this idea is to generate individuals based on a mutation-based breeding method (see Subsection 4.1). The out-breeding panel is created, which allows the user to choose images by a dissimilarity filter (see Subsection 4.2). Evolution can be implemented with drag-and-drop mating.

\subsection{Mutation-Based Breeding}

Each gene in the gene pool that is set in the initial population represents one feature abstracted from classical Batik patterns. In the evolutionary process, the search space is changed by introducing new genes applied by mutation. The new genes are generated by mutating one current population in two ways.

1) Gaussian mutation: add a random value from a Gaussian distribution to every point in unit set of all the genes to create a new offspring. For example, if a genotype $G(i)$ consists of two genes, the mutation operator will generate a Gaussian random number $r$ and apply it in sequence to every point as shown in Fig.7.

2) Style mutation: randomly select a different value for parameter $C_{i}$ in each allele to change the style of the line that joins two points in every allele of unit sets. For example, a number of styles have been applied like straight line, oval, curves, etc.

\subsection{Dissimilarity Filter}

As we stated, the goal of this mechanism is to generate populations that are quite different from the one selected in the evolving panel. To implement this idea, we need to develop an approach to compare images, and keep the most dissimilar ones by filtering out the similar ones produced by the mutation breeding method.

In order to compare two images, it is useful to associate an energy or essential quality to an image. However, this is an extremely difficult task. In some situations the similarity value might be small, but the visual error is huge. So one difficulty is to find a good way to measure the visual difference that "agrees with our eyes".

We present a similarity metric based on the singular value decomposition (SVD) ${ }^{[17]}$. In our approach, we begin with an $m \times n$ matrix $\boldsymbol{A}$ to represent our image. The SVD of matrix $\boldsymbol{A}$ can be expressed as,

$$
\boldsymbol{A}=\boldsymbol{U} \boldsymbol{\Sigma} \boldsymbol{V}^{\mathrm{T}}=\sigma_{1} U_{1} V_{1}^{\mathrm{T}}+\sigma_{2} U_{2} V_{2}^{\mathrm{T}}+\cdots+\sigma_{r} U_{r} V_{r}^{\mathrm{T}},
$$

where $\boldsymbol{U}$ and $\boldsymbol{V}$ are orthogonal matrices, $\boldsymbol{U}$ is $m \times m$ and $\boldsymbol{V}$ is $n \times n . \quad \boldsymbol{\Sigma}$ is rectangular with the same dimensions as $\boldsymbol{A}$, the diagonal entries of which is $\sigma_{i}$, and is called singular values of $\boldsymbol{A}$, where $r=\min (m, n)$, with $\sigma_{1} \geqslant \sigma_{2} \geqslant \cdots \geqslant \sigma_{r} \geqslant 0$. Any large-scale features in the image will be reflected by redundancy in the columns or rows of pixels, and thus we hope to recapture these features in an approximation by a matrix of lower rank than $r$. We form the feature vector $\boldsymbol{f}_{i}$ as

$$
\boldsymbol{f}_{i}=\left(\sigma_{1}, \sigma_{2}, \ldots, \sigma_{k}\right), \quad 1 \leqslant k \leqslant r
$$

where $\sigma_{i}$ is the $i$-th singular value in $A$.

Then we define the Frobenius norm of the feature $\boldsymbol{f}_{i}$ as

$$
\|\boldsymbol{I}\|_{\text {Fro }}=\sqrt{\sum_{i=1}^{k}\left|\boldsymbol{f}_{i}\right|^{2}} .
$$

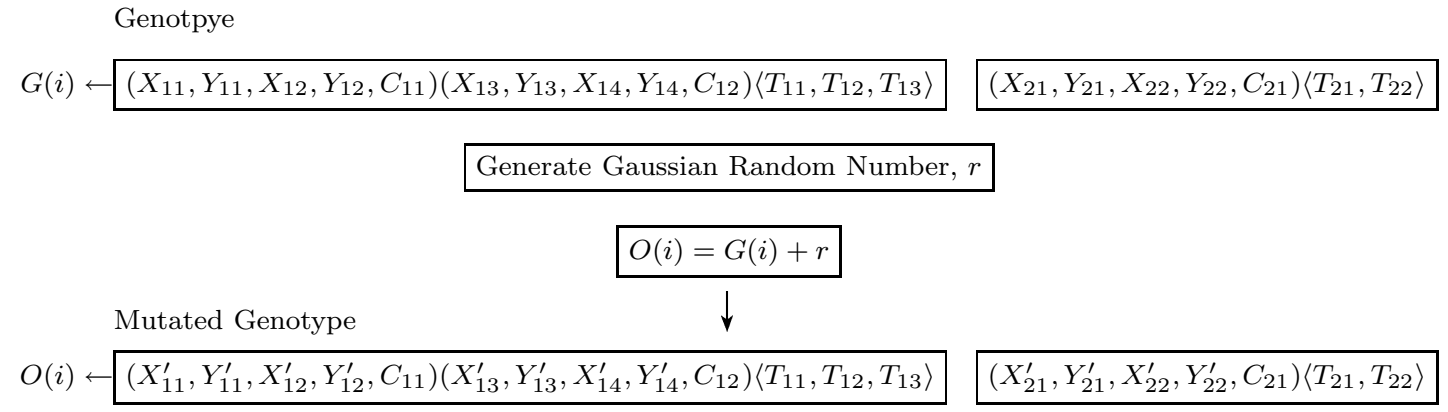

Fig.7. Example of Gaussian mutation. 
The dissimilarity between image $a$ and its alteration $a^{\prime}$ is given by the following formula:

$$
\operatorname{dissim}_{a, a^{\prime}}=\frac{\left\|\boldsymbol{I}_{a}-\boldsymbol{I}_{a^{\prime}}\right\|_{\text {Fro }}}{\left\|\boldsymbol{I}_{a}\right\|_{\text {Fro }}} .
$$

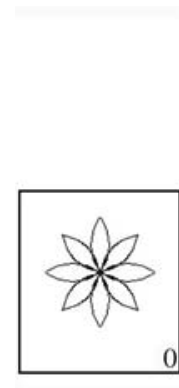

(a)

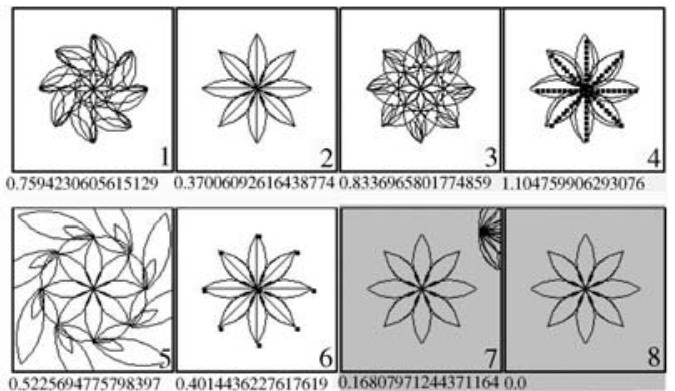

(b)
Fig.8. (a) Original parent. (b) Mutated populations with dissimilarity.

An example is given in Fig.8. Image 0 is selected from the evolving panel, the other eight are the subset of the images generated by the breeding method. Here, the dissimilarities between the mutation populations and image 0 are shown below. Accordingly, the dissimilar order list of these images is: $\{4,3,1,5,6,2,7,8\}$. This order seems to match approximately what we get from our eyes. The only inappropriate one is that individual 4 is considered more dissimilarity than individuals 3 and 1 . The dissimilarity filter is used to select $75 \%$ from the mutation population and render them by the out-breeding panel. In this case, images 7 and 8 are eliminated by the filter, while the rest six will be applied to the breeding panel. We find empirically that most of the populations generated by this mechanism appear to be more elaborate and attractive than the original one. The out-breeding mechanism has been very effective in searching for innovative patterns through maintaining the diversity in exploring the design space.

\section{Description of the System}

\subsection{Framework of the Evolutionary Art System for Batik Design}

The overall architecture of our system is given in Fig.9. Two main modules are included in the system: a Gene Generator is used to generate new patterns. An IEA Engine drives the evolution.

The Gene Generator (see Subsection 5.2) applies predefined genes to the gene pool, which is the basic step for initialization. The mathematic model for Batik is used to produce traditional Batik features and offer genes representing conventional patterns. The users could submit new genes to the gene pool in the evolutionary process from the IEA engine. Therefore the generated genes could be preserved for the new rounds of evolution.

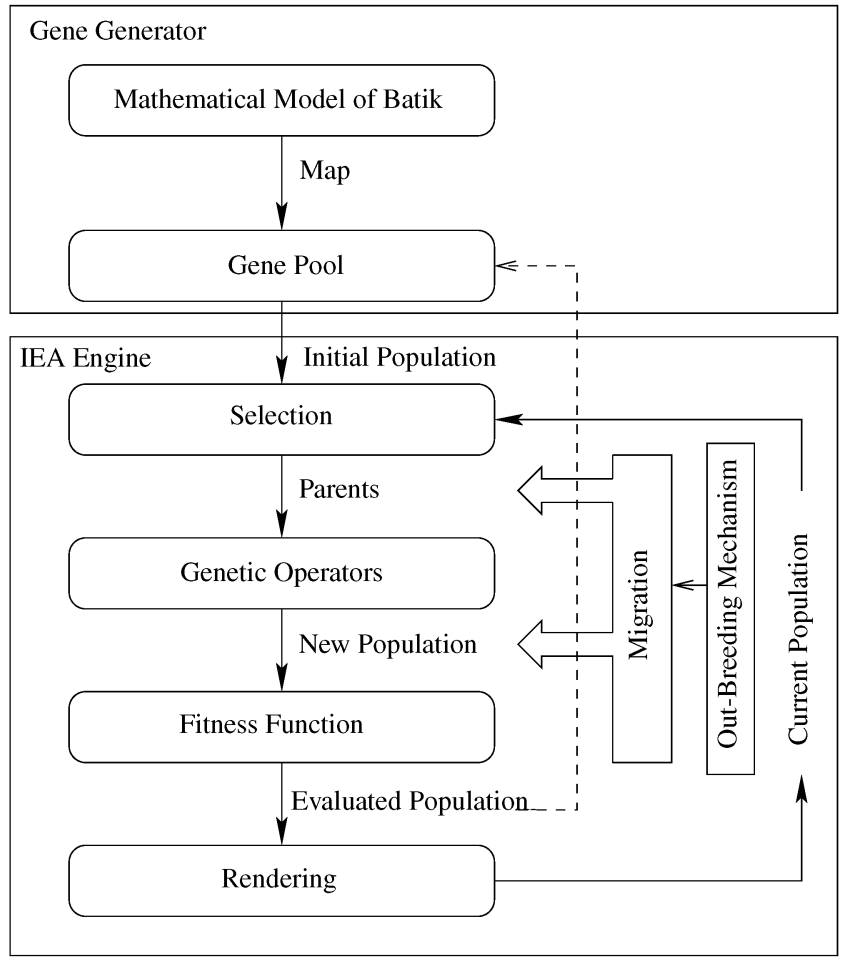

Fig.9. Framework of our evolutionary art system.

In the IEA engine, which will be presented in Subsection 5.3, the user plays a key role in the evolutionary process. The genetic representation, definition of the corresponding operators and an out-breeding mechanism are substantially different from all existing EASs.

As we mentioned before, two new contributions are introduced in our EAS. First, the compact genotype is created to represent Batik patterns. It is composed of a number of genes, each of which could generate one feature in a pattern, and various features form the final design. Second, the out-breeding mechanism is presented in addition to the normal steps in an IEA process. This mechanism uses a similarity metric to evolve dissimilar patterns, in order to deal with stagnation during the evolution.

\subsection{Gene Generator}

In our system, predefined genes stored in the gene pool are used to seed the initial populations. This method reduces the processing time spent in the evolutionary process and overcomes some limitations in searching in an unbounded design space. Two methods are introduced to generate new genes for the gene pool.

First, a mathematical model is used to generate genes by several parameters. A quintuple form, 
( $D, S, T$, Trans, $N)$, as the input of the model, will be converted into the corresponding gene. In this form, $D$ is the angle formed by the growing motif edges with the vertical axis. $S$ controls the length of the path that emanates from the starting point to the end point. $T$ represents how the two points connect with each other, e.g., straight line, curve, oval, etc. Trans, the normal geometric transformation, maps the minimal set of points and edges into the gene. $N$ is the number of times to use the transformation to reach the nonredundant set. Fig.10 shows some genes generated by our model and also includes the corresponding input parameters.

Second, the users could submit new genes to the gene pool in the evolutionary process. Therefore new genes are preserved temporarily in the gene pool for the next round of evolution. As a result, the space for generating initial populations is enlarged.

The gene generator also helps designers to understand what is included in a good design. All the good features expressed by these genes merge together to form the final design. The genes produced by the gene generator contain the basic elements like specific curve which we found in most classic Batik patterns. By analysing the evolved Batik designs and their genes, we could gain some insight as to what genes have played an important role in good designs. We can also use such analysis to understand different preferences by different users.

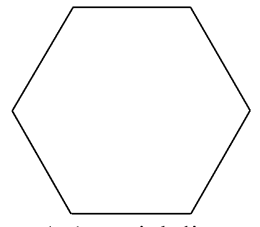

1,1 , straightline, rotate, 6

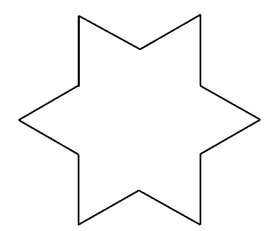

2,1 , straightline, rotate, 6

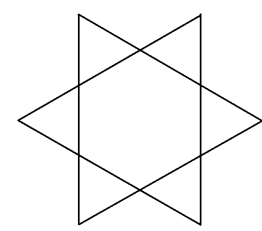

2, 2, straightline, rotate, 6
Fig.10. Examples of genes generated by our model.

\subsection{IEA Engine}

IEA is employed to drive the evolution. The main steps of the evolutionary process are described as follows.

\subsubsection{Initialization}

Gene generator assists the IEA engine to generate more genes for the gene pool. The gene pool is a gene repository, in which genes are combined to form the initial genotypes. When initialization is started, genes are randomly chosen from the gene pool, normally no more than three genes will be used in a genotype in our system.

\subsubsection{Selection}

Selection is manipulated by users. Our goal in this stage is to explore new evolutionary paths, and to increase population diversity thus avoiding the loss of interest by the user in addition to searching what the user is interested in. It is achieved by two methods, migration and out-breeding mechanism. Migration allows a user to combine the current population with the previously created images. This is implemented by a migrating archive, where images are transferred and saved till the end of the process. During the evolution, we can, for instance, preserve the designs that we want to evolve later by dragging it into the archive. This feature allows a user to recall previous designs, while it is also possible to transfer current designs to the previous process. This is extremely useful for overcoming the existing weakness in exploring the design space.

Out-breeding mechanism is the new approach presented in our system, which is described in Section 4. It introduces new populations when the user is stuck in the evolution. Users choose one individual from the current population, and then its alterations will be produced by out-breeding mechanism. Any images shown in this panel could be selected and dragged to the evolving panel to continue evolution.

\subsubsection{Genetic Operators}

The genetic operators are aimed to reproduce a new set of individuals, to maintain diversity, and to evolve towards individuals that the user prefers. According to our representation, we use two kinds of operators: crossover and mutation.

Two kinds of crossover operators are used. Examples of crossover are shown in Fig.11.

1) Gene swap: randomly select points from parents, and then reproduce new offspring by using linear combinations of genes. Both single-point and multiple-point crossover are covered.

2) Gene replacement: randomly select genes from different parents, and then crossover these genes to generate new ones which then replace the old ones.

- Exchange two attributes in genes to generate new ones.

- Merge the maps of points and edges into a new map, one-point crossover is applied to the vector of transformation.

Mutation operator is applied by randomizing the order of its genes and modifying the values according to the mutation rate in each allele.

For example, the system reproduces a new set of individuals using two parents which are shown in Fig.12(a). New sets of offspring generated by the genetic operators are the eight images in Fig.12(b). 


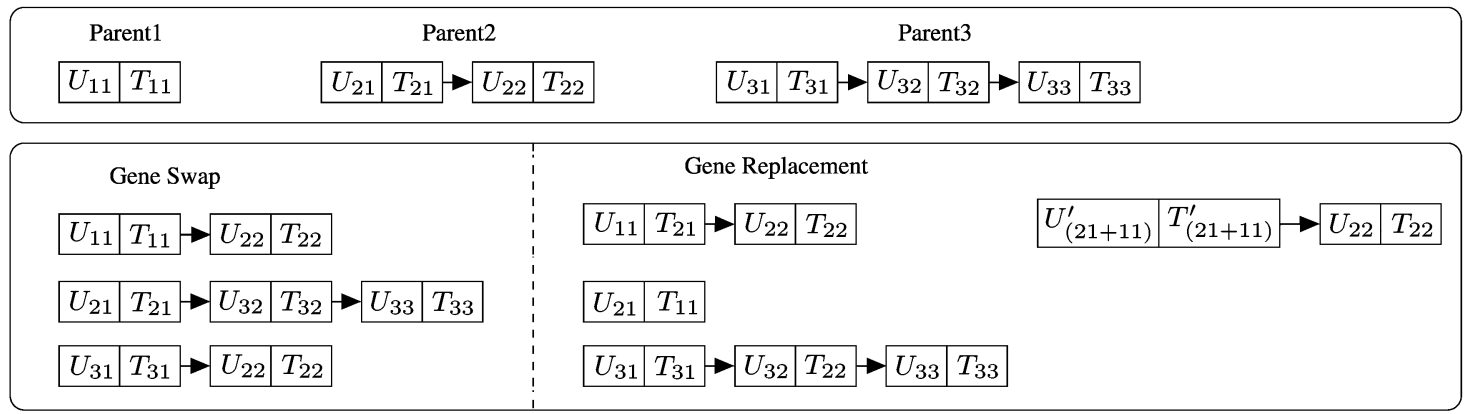

Fig.11. Examples of crossover.

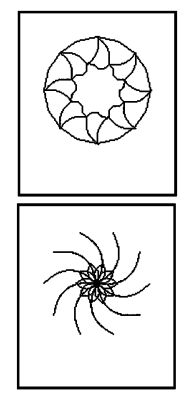

(a)

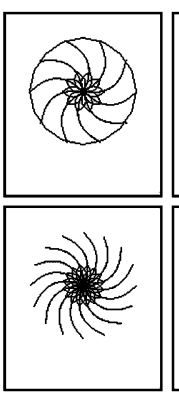

Fig.12. (a) Parents.

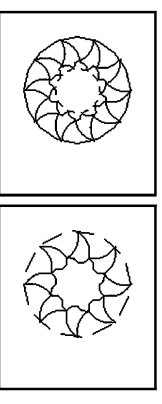

(b)

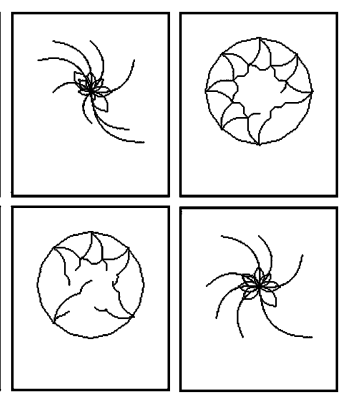

\subsubsection{Fitness Function}

The user evaluates all the output of the system, and interacts with it to choose the better ones as the input to the next generation. This operation is iterated until the user obtains a satisfactory image.

\section{Experimental Studies}

\subsection{System Development for Batik Design}

We set up an online interactive EAS for creating Batik design. The user interface shares some similarity to other systems (especially $[6,11]$ ).

Initial populations generated randomly by the gene pool are displayed on the evolving panel. Table 3 shows parameters for the initial 15 genes which are used in the gene generator. From the displayed set the user selects more than one drawings as parents. The mating and mutation operations are then applied to them to produce a set of progeny patterns. This process is repeated multiple times to "evolve" a drawing of interest to the user. It is used to produce the final Batik-like pattern which will be shown in a new window.

Two external panels, migration panel and outbreeding panel, are also used during the evolutionary process. Evolved patterns may be saved to the migration panel and later recalled for mating with other evolved patterns. The out-breeding panel is an option for the user to choose interesting patterns that are very
Table 3. Parameters for the Initial 15 Genes

\begin{tabular}{cccllr}
\hline Gene & $D$ & $S$ & $T$ & Trans & $N$ \\
\hline 1 & 6 & 1 & oval & rotation & 10 \\
2 & 4 & 1 & oval & rotation & 8 \\
3 & 1 & 1 & curve & rotation & 5 \\
4 & 2 & 1 & straight line & rotation & 5 \\
5 & 1 & 1 & curve4 & rotation & 5 \\
6 & 3 & 1 & oval & translation & 5 \\
7 & 1 & 1 & straight line & translation & 5 \\
8 & 1 & 1 & straight line & rotation & 4 \\
9 & 1 & 1 & straight line & rotation & 3 \\
10 & 3 & 2 & curve & rotation & 8 \\
11 & 4 & 4 & curve4 & rotation & 10 \\
12 & 4 & 1 & dot10 & rotation & 8 \\
13 & 2 & 1 & dot0 & rotation & 8 \\
14 & 1 & 1 & straight line & rotation & 16 \\
15 & 6 & 6 & curve4 & rotation & 10 \\
\hline & & & & &
\end{tabular}

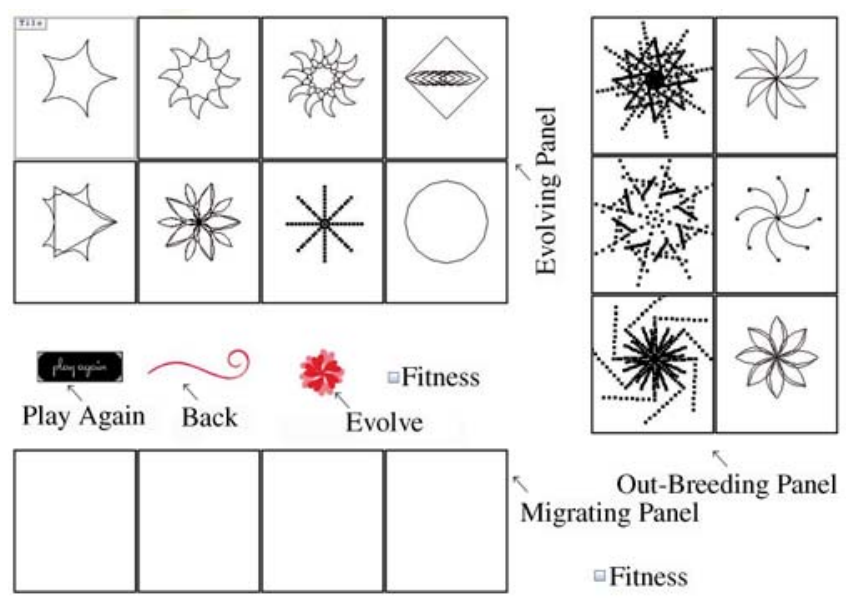

Fig.13. Main window of our EAS.

different from the evolving ones to the evolving panel.

The main window of the system is shown in Fig.13. The drawings rendered in the out-breeding panel are variations of the third one in the second row of the current populations.

In interactive evolution, a user usually does not quite know in advance what they are looking for. He/She 
might want to backtrack. For this reason, our system includes a Back button that allows one to revisit previous generations. Play again button is also provided for the user to restart the process from random initial populations. In order to tessellate the motif into Batik design, Tile is added on the pattern in the evolving panel, on which the user's mouse is focused.

\subsection{Evolved Batik Patterns}

At the application level, the goal of our system is to generate Batik-like artworks that satisfy user's preferences.

How to construct Batik motifs is one of the most important aspects in designing Batik. Batik is a painting or a form of writing on cotton cloth, applied with the aid of a tool called cap, each of which makes up a design unit $^{[3]}$. Batik in forms of various motifs have specific characteristics which are passed onto cloth.

Some of the traditional design could be produced by the mathematical model of Batik as the initial populations. In Fig.14, the top three patterns are the realworld Batik, while the three at the bottom are produced from the gene pool as initial populations, which seem to capture some features in Batik very well.
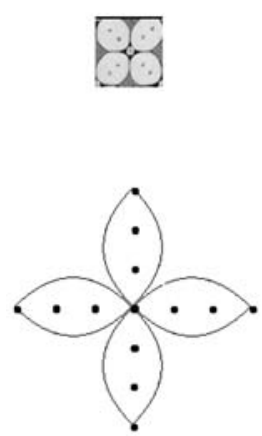

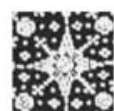

(a)

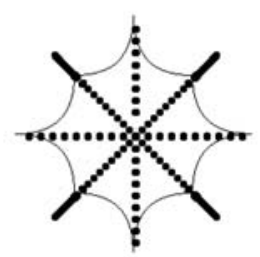

(b)
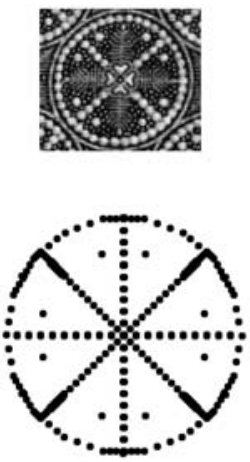

flowers or animals, are incorporated to compose this particular pattern. Examples of this category created by our system are shown in Fig.17.

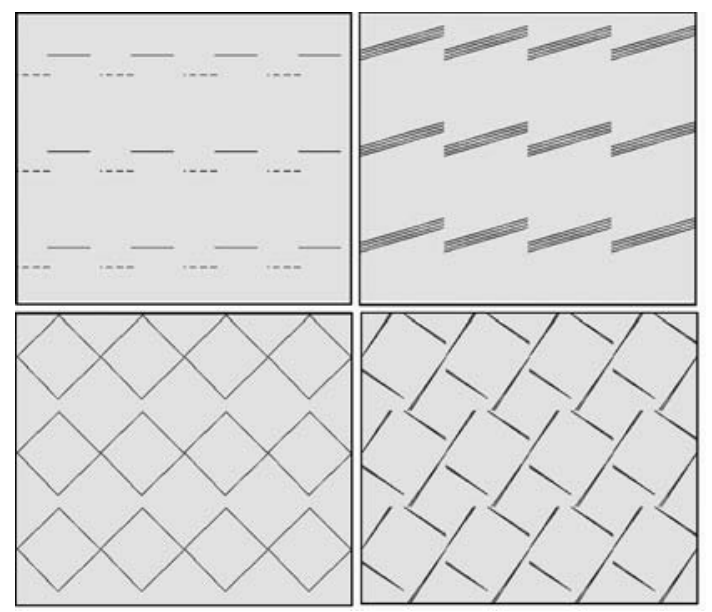

Fig.15. Klowong pattern generated by our system.

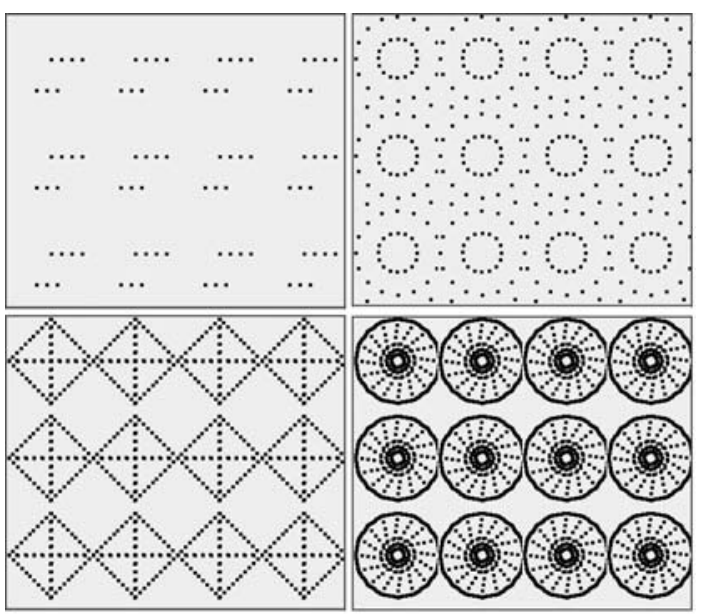

Fig.16. Cecek pattern generated by our system.

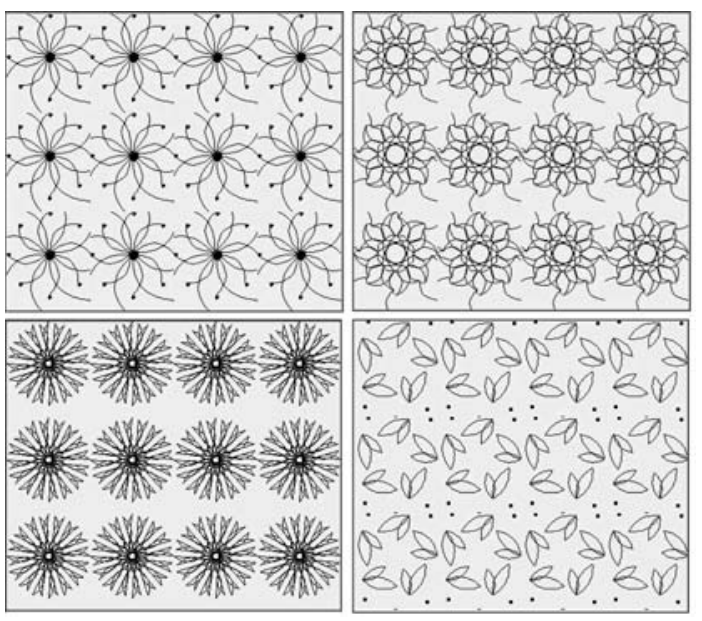

Fig.17. Vegetation pattern generated by our system.

Fig.14. (a) Three similar real-world Batik patterns. (b) Initial individuals generated by the mathematical model.

Although there are thousands of different Batik designs, particular artworks have traditionally been associated with festivals and specific religious. Various forms of motifs can be identified as Batik design other than any other patterns. Here three different categories of traditional Batik features have been recognized in the patterns that are generated by our system.

1) Klowong: different kinds of lines form this kind of patterns. Fig.15 shows the patterns generated by our system.

2) Cecek: various dots are used to construct this type of design, see Fig.16.

3) Vegetation: naturalistic forms, such as leaves, 
However, these examples of motifs are not limited to particular symmetric patterns. Other innovative Batik design can also be developed using our system.

\subsection{Efficiency of Our Interactive System}

We have conducted five experiments using our system, each of which runs twice, with and without the out-breeding mechanism. This was done by using Back button from the same initial populations. In the setting for our experiments, 15 genes (see Table 3 ) were used in the gene pool, and no more than 2 genes were combined to generate the initial individuals. The population size is constrained to 8. In order to show the efficiency of our system, three factors are analyzed, i.e. the user's time, the average number of fitness evaluations by users in every generation and the advantages compared with other IEC systems.

Table 4. Performance Evaluation

\begin{tabular}{llll}
\hline Mode & $\begin{array}{l}\text { Time- } \\
\text { consumption }\end{array}$ & $\begin{array}{l}\text { Number of } \\
\text { generations }\end{array}$ & $\begin{array}{l}\text { Number of } \\
\text { evaluated } \\
\text { individuals }\end{array}$ \\
\hline Handmade & $30^{\prime}$ & $\times$ & $\times$ \\
IEC & $9^{\prime} 21^{\prime \prime}$ & 17.6 & 140.8 \\
IEC (out-breeding & $5^{\prime} 2^{\prime \prime}$ & 7.8 & 109.2 \\
mechanism) & & & \\
\hline
\end{tabular}

Table 4 shows the average time consumption, the average number of generations, and the average number of fitness evaluations made by a human user and our system (with and without the out-breeding mechanism) over the five experiments. Clearly, our system took only $1 / 3$ of the time used by the human user, without resorting to the out-breeding mechanism. With out-breeding mechanism, the time is further reduced by half. For IEC with the out-breeding mechanism, the total numbers of generations and evaluations are also fewer than those of IEC without it. Although the number of individuals for every generation is increased because of the mechanism, the total number of generations is reduced because it takes less steps to find a satisfactory design. Although more rigorous and more comprehensive evaluation of our system is needed, our preliminary study here does illustrate the efficiency and effectiveness of our system.

To evaluate our system further, we focus on one experiment to compare the systems with and without the out-breeding mechanism. A user may assign a value between 0 and 10 to the fitness of every individual during the evolutionary process. Fig.18 shows the average fitness of all the individuals generated by the system with and without the out-breeding mechanism. It can be seen from Fig.18 that the system with the out-breeding mechanism can improve the fitness significantly faster than the system without it, especially between the 2nd and 6th generation. It is worth noting that the fitness was assigned by the user, so a higher fitness value does reflect the user's satisfactory level with the evolved patterns. Similar conclusions can be drawn from other experiments.

Fig.19 shows the initial populations used in our experiment. Figs. 20 and 21 show the populations at the

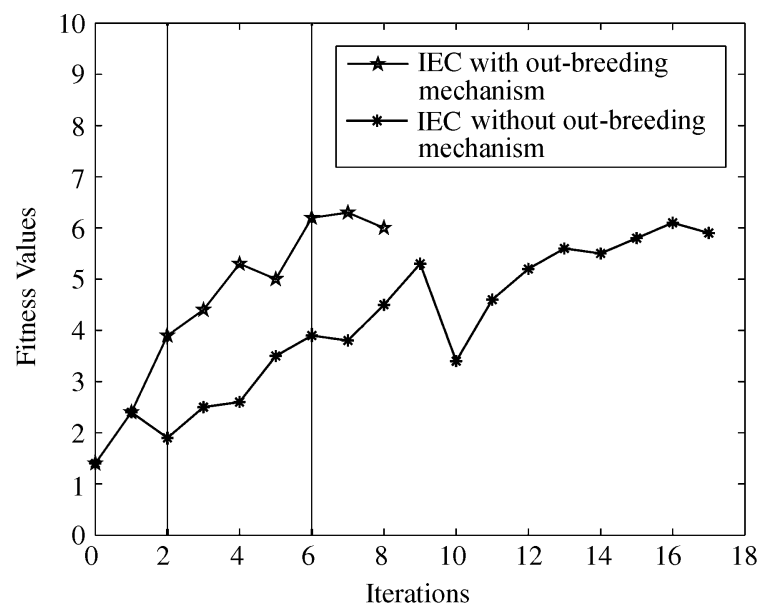

Fig.18. Average fitness values along with evolutions. The second and sixth iterations are the steps when the individuals from the out-breeding mechanism are introduced.

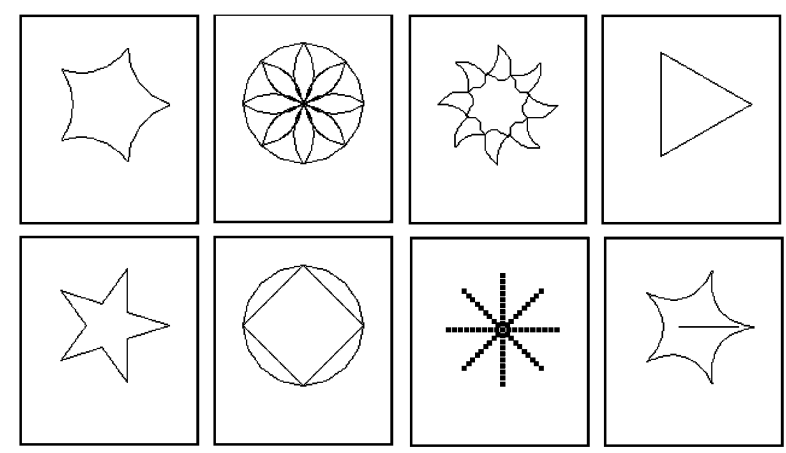

Fig.19. Initial populations in our experiments.
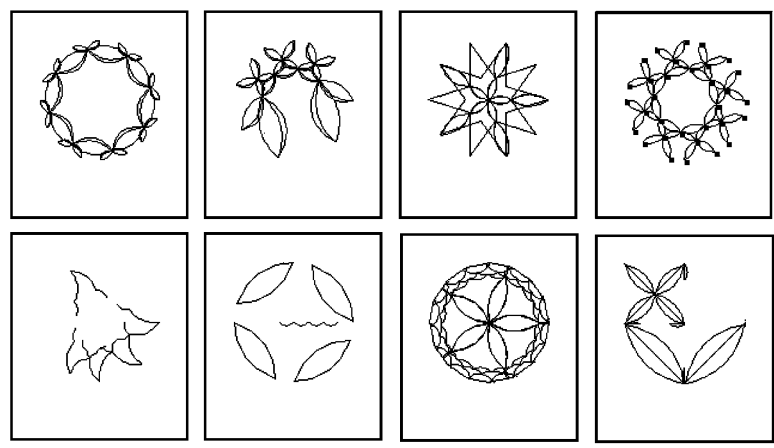

Fig.20. Populations in the 6th generation without the outbreeding mechanism. 

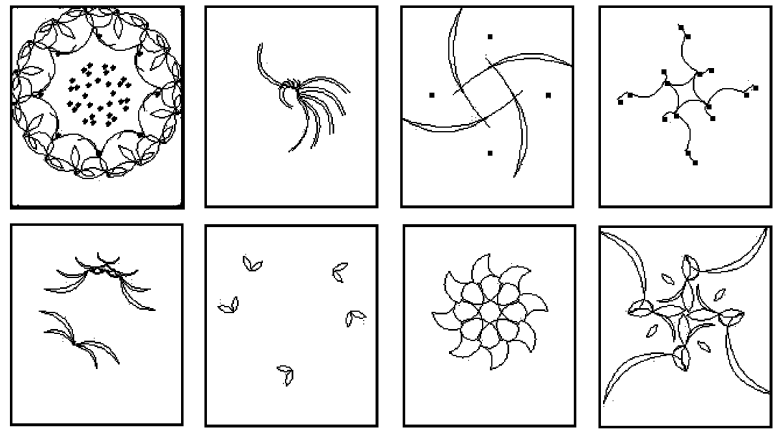

Fig.21. Populations in the 6th generation with the out-breeding mechanism.

6 th generation of our systems without and with the out-breeding mechanism respectively. It is interesting to observe that many individuals in Fig.21 (with the out-breeding mechanism) have evolved patterns that are quite different from those in the initial population.
Asymmetric patterns have also been evolved. Figs. 22 and 23 show the evolved Batik design by tiling the evolved patterns. Note that diagonal tessellations are generated in the latter one in addition to horizontal and vertical tessellations, which are characteristics in traditional Batik.

In comparison with other IEC systems ${ }^{[19]}$, our system has several advantages. First, our system has a unique encoding method that enables us to gain insight into the relationship between genotypes and phenotypes much better. It is feature-based, not pixelbased. Second, the out-breeding mechanism enables us to explore the design space effectively and facilitate the evolution of innovative designs. Third, our system has a rich set of crossover and mutation operators that encourage effective search in the design space. Table 5 summarises some of the major differences and similarities between our system and other IEC systems.
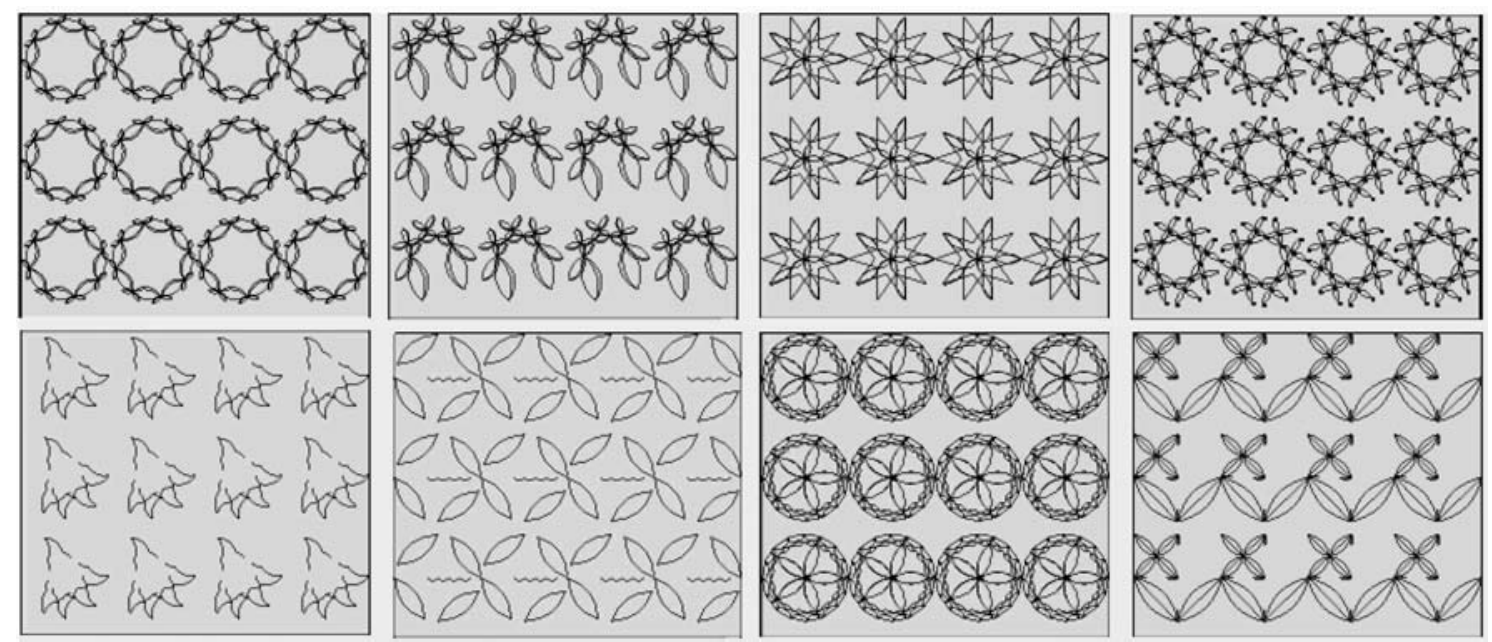

Fig.22. Tessellation of the populations in the experiment without the out-breeding mechanism.
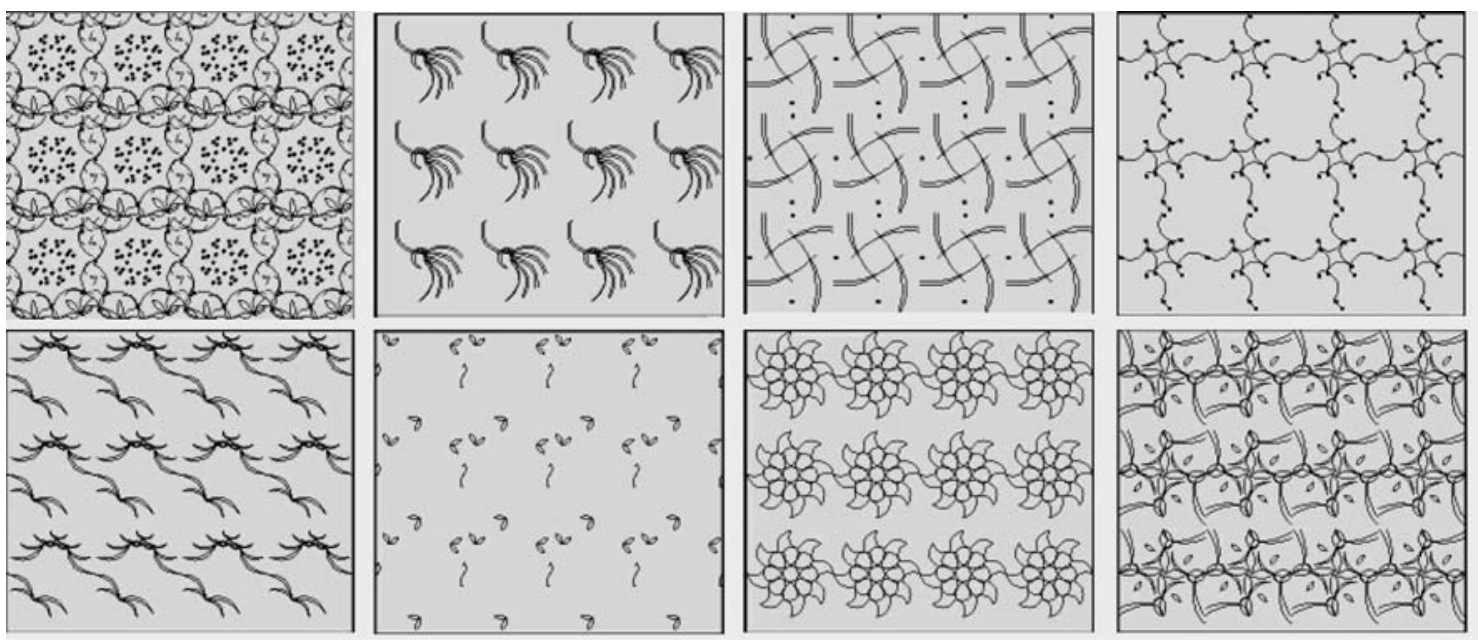

Fig.23. Tessellation of the populations in the experiment with the out-breeding mechanism. 
Table 5. Features of Our System and Other IEC Systems

\begin{tabular}{lcc}
\hline & Our System & Other IEC Systems \\
\hline Coding & Feature-based & Pixel-based \\
Rating levels & 2 & $5 / 10 / 100$ \\
Population size & 8 & $10 / 20$ \\
Number of & $\geqslant 2$ & $1 / 2$ \\
parents & & \\
Out-breeding & $\sqrt{ }$ & $\times$ \\
mechanism & & $\sqrt{ } / \times$ \\
Migration & $\sqrt{ }$ & \\
\hline
\end{tabular}

However, cautions should be taken in drawing too strong conclusions because we have conducted only limited experiments so far. More rigorous and large-scale evaluation of our system is needed.

\section{Conclusions and Future Work}

This paper introduces a novel evolutionary design system for Batik design. The system has incorporated several new ideas in evolutionary design, which enable the system to perform significantly better than existing IEC systems. The two most important new ideas are the representation of Batik patterns and the outbreeding mechanism. The new representation is based on features, not pixels, of a Batik pattern. It can demonstrate the link between good designs and genes clearly, which can give insight into good Batik designs. The out-breeding mechanism can help the system to avoid stagnation in perceived local optima and explore innovative designs that are very different from what a user has in mind initially.

We have carried out a number of empirical studies to evaluate the performance of our system. Our preliminary results have confirmed the effectiveness and efficiency of our system in comparison with other IEC systems. The future work of this research includes several major tasks. First, we would like to make our system available online so that everyone can use it and evaluate its strength and weakness. Second, we would like to study alternative similarity measures to that in Subsection 4.2 in order to improve our system further. Third, we would like to study automatic fitness evaluation methods by learning from both human users and previous generations in the evolution.

\section{References}

[1] Romero J, Machado P. The Art of Artificial Evolution: A Handbook on Evolutionary Art and Music. Springer Neitherlands, 2008.

[2] Bentley P. An Introduction to Evolutionary Design by Computers. Evolutionary Design by Computers, Bentley P J (ed.), San Francisco, CA: Morgan Kaufmann Publishers, 1999, pp.1-73.
[3] Kerlogue F, Zanetini F. Batik: Design, Style and History. London: Thames and Hudson, 2004.

[4] Schnier T, Yao X, Beale R, Hendley R, Byrne W. Nature inspired creative design - Bringing together ideas from nature, computer science, engineering, art, design. In Proc. the Seventh International Conference on Adaptive Computing in Design and Manufacture (ACDM 2006), Bristol, UK, April 25-27, 2006, pp.237-240.

[5] Koza J. Genetic Programming: On the Programming of Computers by Means of Natural Selection. Cambridge, MA: MIT Press, 1992.

[6] Sims K. Artificial evolution for computer graphics. In Proc. the 18th Annual Conference on Computer Graphics and Interactive Techniques (SIGGRAPH 1991), New York: ACM Press, 1991, pp.319-328.

[7] Poli R, Cagnoni S. Genetic programming with user-driven selection: Experiments on the evolution of algorithms for image enhancement. In Proc. the 2nd Annual Conf. Genetic Programming, Standford, USA, Morgan Kaufmann, July 13-16, 1997, pp.269-277.

[8] Wang S F, Wang S, Takagi H. User fatigue reduction by an absolute rating data-trained predictor in IEC. In Proc. IEEE Congress on Evolutionary Computation, Vancouver, Canada, July 16-21, 2006, pp.2195-2200.

[9] Lutton E. Evolution of Fractal shapes for artists and designers. International Journal on Artificial Intelligence Tools, 2006, 15(4): 651-672.

[10] Kicinger R, Arciszewski T, De Jong K A. Evolutionary computation and structural design: A survey of the state of the art. Int. J. Computers and Structures, 2005, 83(23/24): 1943-1978.

[11] Dawkins R. The Blind Watchmaker. Harlow Longman, 1986.

[12] Coates P, Broughton T, Jackson H. Exploring threedimensional design worlds using Lindenmayer systems and genetic programming. Envolutionary Design by Computers, Bentley P J (ed.), San Mateo, CA: Morgan Kaufmann, 1999, pp.323-342.

[13] Hornby G S. Generative representation for evolutionary design automation [Ph.D. Dissertation]. Department of Computer Science, Brandeis University, Waltham, USA, 2003.

[14] Rooke S. Eons of genetically evolved algorithmic images. Creative Evolutionary Systems, Chapter 13, Bentley P J, Corne D W (eds.), San Francisco: Morgan Kaufmann, 2001, pp.339365.

[15] Machado P, Cardoso A. NEvAr - The assessment of an evolutionary art tool. In Proc. the Symposium on Creative \& Cultural Aspects and Applications of AI \& Cognitive Science (AISB 2000), Birmingham, UK, April 17-20, 2000, pp.219229.

[16] Secretan J, Beato N, D'Ambrosio DB, Rodriguez A, Campbell A, Stanley K O. Picbreeder: Evolving pictures collaboratively online. In Proc. Computer Human Interaction Conference, ACM, New York, 2008, pp.1759-1768.

[17] Wang Y, Tan T, Zhu Y. Face verification based on singular value decomposition and radial basis function neural network. In Proc. the 4th Asian Conference on Computer Vision, Taipei, China, Feb. 27-Mar.3, 2000, pp.432-436.

[18] Walker J, Miller J. Improving the evolvability of digital multipliers using embedded Cartesian genetic programming and product reduction. In Proc. the 6th International Conference (ICES 2005), Sitges, Spain, Sept. 12-14, 2005, Springer, pp.131-142.

[19] Takagi H. Interactive evolutionary computation. In Proc. the 5th International Conference on Soft Computing and Information/Intelligent Systems, Iizuka, Japan, October 1998, pp.41-50. 


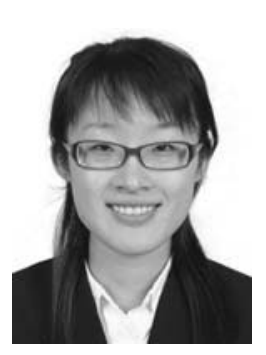

Yang Li received the B.Sc. degree from the School of Information Engineering, University of Science and Technology Beijing, China, in 2004, where she is also currently working toward the Ph.D. degree. She was a visiting Ph.D. student at the Centre of Excellence for Research in Computational Intelligence and Applications (CERCIA) at the University of Birmingham, UK, from October 2007 to September 2009. Her research interests include data integration, semantic web, interactive evolutionary computation and realworld applications of evolutionary algorithms.

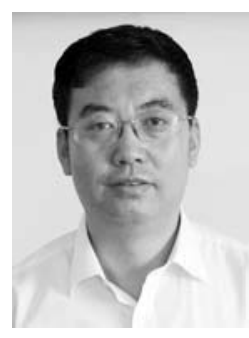

Chang-Jun $\mathbf{H u}$ received the Ph.D. degree from Peking University, Beijing, China, in 2001. He is currently a professor at the School of Information Engineering at the University of Science and Technology Beijing, China. His main research interests include parallel computing, parallel compilation technology, parallel software engineering, network storage system, data engineering and software engineering.

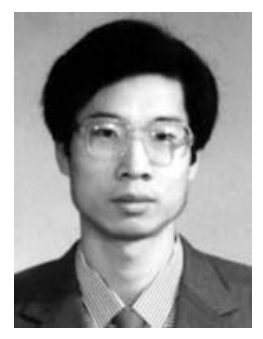

Xin Yao received the B.Sc. degree from the University of Science and Technology of China (USTC), Hefei, in 1982, the M.Sc. degree from the North China Institute of Computing Technology, Beijing, in 1985, and the Ph.D. degree from USTC in 1990. He worked as an associate lecturer, lecturer, senior lecturer and associate professor in China and later on in Australia. Currently, he is a professor of computer science at the University of Birmingham (UK), a visiting chair professor at the USTC and the director of the Centre of Excellence for Research in Computational Intelligence and Applications (CERCIA). He was the editor-in-chief of the IEEE Transactions on Evolutionary Computation (2003-2008), an associate editor or editorial board member of 12 other journals, and the editor of the World Scientific Book Series on Advances in Natural Computation. His major research interests include evolutionary computation and neural network ensembles. He was awarded the President's Award for the Outstanding Thesis by the Chinese Academy of Sciences for his $\mathrm{Ph}$.D. work on simulated annealing and evolutionary algorithms in 1989. He won the 2001 IEEE Donald G. Fink Prize Paper Award for his work on evolutionary artificial neural networks. He is a fellow of IEEE and a distinguished lecturer of the IEEE Computational Intelligence Society. 